\title{
Psychological Well-Being, Self-Reported Physical Activity Levels, and Attitudes to Physical Activity in a Sample of New Zealand Adolescent Females
}

\author{
Daniel Shepherd*, Chris Krägeloh, Clare Ryan, Grant Schofield \\ School of Public Health and Psychosocial Studies, Faculty of Health and Environmental Sciences, \\ Auckland University of Technology, Auckland, New Zealand \\ Email: \{'daniel.shepherd, chris.krageloh, clarya07, grant.schofield\}@aut.ac.nz
}

Received January $26^{\text {th }}, 2012$; revised February $27^{\text {th }}, 2012$; accepted April $1^{\text {st }}, 2012$

\begin{abstract}
Physical activity (PA) is a key component of healthy development, not only physically but also psychologically. The aim of the present study was to measure PA levels and psychological well-being in adolescent females using a cross-sectional design, and to investigate the relationship between the two. Psychological well-being (self esteem and lack of depression, anxiety, and stress), PA, and established predictors of PA from the Theory of Reasoned Action (Fishbein \& Ajzen, 1975; Madden, Ellen, \& Ajzen, 1992; health consciousness, significant others, priority, perceived barriers, and attitudes) were measured using 148 adolescent females aged 16 to 18 years. Results show a link between depression and level of PA, and between anxiety and PA. Attitudes towards PA, priority of PA, and perceived barriers to PA were also related to levels of PA. However, there were no significant associations between psychological well-being and attitudes towards PA, even though psychological well-being may influence the actual level of activity. Positive associations between PA and psychological well-being in adolescent females encourage future studies into causal relationships between the two. The most effective strategies for increasing PA in middle to late adolescent females may be targeting perceived barriers to PA rather than attitudes.
\end{abstract}

Keywords: Adolescent Health; Physical Activity; Psychological Well-Being; Attitudes; Barriers; Theory of Reasoned Action

\section{Introduction}

Existing data indicate good levels of physical activity (PA) by most New Zealand children, though there is a definite reduction in participation in adolescence (defined here as 12 to 18 years old), especially for females (Riddoch \& Boreham, 1995; Sallis, Prochaska, \& Taylor, 2000; Schofield, Mummery, Schofield, \& Hopkins, 2007). During mid to late adolescence, females are at increased risk of inactive lifestyles when compared with male youth (Hohepa, Schofield, \& Kolt, 2004; Schofield et al., 2007; SPARC, 2003). This is concerning as adolescence is an important age for girls to stay active, and PA levels often track into adulthood (Kahn et al., 2008; Robbins, Sikorskii, Hamel, Wu, \& Wilbur, 2009; Schofield et al., 2007). Developmentally, adolescence is characterized by rapid psychological, psychosocial and physical change. The onset of puberty coincides with unprecedented social demands which often translate into significant psychological stressors, and females are more likely to have a more negative body image and lower selfesteem than males. PA may buffer the negative impacts of puberty, and sustain psychological and physical well-being during and beyond adolescence (Dugan, 2008).

Increasing PA in adolescent females would lead to better population health, lower health care costs, and a reduction in social inequalities in health (Dugan, 2008). To this end substantial effort has been directed into identifying the barriers that adolescents face when attempting to undertake PA (Robbins et al., 2009). Barriers to PA reported by adolescent New Zealand

*Corresponding author. females included a dislike for performing activities in front of other class members singularly, perceived incompetence, and perceptions of peer judgment, all of which inhibit involvement in physical education classes and attending trials for sport teams (Hohepa, Schofield, \& Kolt, 2006). Additionally, research on social influences and adolescent PA, although somewhat equivocal, suggests that coaches, teachers, siblings, parents, and peers all play a role in shaping PA levels during adolescence. For example, friends may be an important influence (Seabra, Mendonça, Thomis, Peters, \& Maia, 2008), especially among adolescent girls (Schofield et al., 2007).

The impact of PA on adolescent psychological well-being has yet to be elucidated. Azar, Ball, Salmon, and Cleland (2008) reviewed the effects of $\mathrm{PA}$ intervention studies on depression in young women between the ages of 18 and 35, and found that women who participated in PA were less likely to report depressive symptoms. Additionally, they found that any amount of PA was associated with fewer depressive symptoms when compared with no PA (Azar et al., 2008), supporting arguments against a dose-response relationship between PA and affective state (Dunn, Madhukak, \& O’Neal, 2001).

The formation of positive attitudes towards PA during adolescence may be an important step towards their actual participation in activity. Smoll and Schutz (1980) reported that attitudes towards PA explained $20 \%$ of variance of PA in a sample of 16- to 18-year old youth. Godin and Shephard (1984) found that the attitudes of 13- to 15-year-olds towards PA, their previous experience with PA as such, and their current habits ex- 
plained almost $50 \%$ of variance of participation in PA. However, others (e.g., Thompson \& Humbert, 2003) appeal for more research into understanding the attitudes and behaviors of adolescents regarding PA. A useful framework here is the Theory of Reasoned Action, a theory of attitude-behavior relationships that links attitudes, subjective norms (akin to perceived social pressure), and behavioral intentions to volitional behavior (Fishbein \& Ajzen, 1975; Madden, Ellen, \& Ajzen, 1992), which has also been successfully applied to PA (Biddle \& Mutrie, 2008). Volitional behavior is posited to result from behavioral intentions, which in turn are said to arise out of a combination of a person's attitude toward performing the behavior in question and the perceived social pressure (subjective norm) put upon them to perform that behavior (Sparks, Guthrie, \& Shepherd, 1997).

There have been few studies investigating the association between PA and psychological well-being in adolescent females (Azar et al., 2008). The present exploratory study examined whether levels of PA in a sample of New Zealand adolescent girls are associated with indicators of psychological wellbeing (i.e., depression, anxiety, stress, and self-esteem). Additionally, known predictors of PA in adults, including attitudes to PA, the influence of others, other preferred activities, perceived barriers, and health consciousness, were also examined within the framework of the theory of reasoned action (Fishbein \& Ajzen, 1975; Madden, Ellen, \& Ajzen, 1992).

\section{Methods}

\section{Participants}

Participants were 148 females, aged between 16 to 18 years old, attending a New Zealand all-girls secondary school in Auckland, New Zealand. The school principal was contacted by the researchers and gave permission to conduct this study at the school. Participants were not offered any incentive to participate, and assent was obtained from each prior to the research commencing. Questionnaires were distributed during class time, and participants were asked to read a participant information sheet prior to completing the questionnaires, which included definitions of PA. To minimize effects of peer pressure, individuals were asked to spread out as much as possible across the classroom and were asked to complete the questionnaires without consultation with their peers. The protocol of the study was approved by the Auckland University of Technology Ethics Committee.

\section{Instruments}

Self-Report Physical Activity. The New Zealand Physical Activity Questionnaire-Short Form (NZPAQ-SF; Mackay, Schofield, \& Schluter, 2007; SPARC, 2004) is a paper-and-pen questionnaire that consists of eight items designed to assess levels of PA in the New Zealand population. The NZPAQ-SF returns a final PA score and categorizes individuals according to one of three PA groups: relatively inactive $(<2.5$ hours of activity per week: PAQ1); relatively active $(2.5-4.9$ hours of activity per week: PAQ2); and highly active (5+ hours of activity per week: PAQ3).

Psychological Well-being. The Depression, Anxiety, Stress Scale-42 (DASS-42; Lovibond \& Lovibond, 1995) consists of 42 items, divided into three subscales: depression, anxiety, or stress. The depression subscale corresponds closely to the ac- cepted criteria for mood disorders, while the anxiety subscale corresponds most closely to the symptom criteria for the various anxiety disorders, with the exception of generalized anxiety disorder (Lovibond \& Lovibond, 1995). The DASS-42 consists of statements to which participants respond using a four-point Likert scale, ranging from 0 (Did not apply to me at all) to 3 (Applied to me very much, or most of the time). Additionally, Rosenberg's Self-Esteem Scale (RSES; Rosenberg, 1965), a brief, ten-item, unidimensional measure of global self-esteem, was administered. Responses to the statements contained in the RSES are made on a four-point scale $(0-3)$, ranging from strongly agree to strongly disagree. Total scores computed from the RSES range from 0 to 30, with higher scores indicating higher self-esteem.

Attitudes. The Attitudes Towards Physical Activity Scale (ATPAS; SPARC, 2003) contains 46 statements scored using a five-point Likert scale, and measures influences on PA. The ATPAS probes factors that have been identified to influence PA behavior, and includes subscales representing health consciousness, attitudes towards PA, significant others, priority of, and barriers to PA. The health consciousness variable investigates factors regarding exercising and health, including living a healthy lifestyle, making changes in daily routines in order to prevent health problems, and following recommended activity guidelines. The variable attitudes towards $P A$ examines participant views of PA its advantages. Significant others explores the influences of peers, family, teachers and important others on their intention to engage in PA, while priority of PA measured the priority of engaging in PA by assessing other possible activities participants may prefer to engage in, such as internet use, video games, and watching the television. The sub-scale barriers to PA include lack of energy, costs associated with PA and the availability of facilities, feelings of safety and the efforts associated with being physically active.

\section{Data Analysis}

Data screening and analyses were undertaken using the Statistical Package for the Social Sciences (SPSS, v.17). Analysis commenced with an evaluation of each scale's psychometric properties, including tests for floor and ceiling effects (item means and standard deviations), internal consistency (Cronbach's alpha), and to validate dimensionality (corrected itemtotal correlations and principal components analysis). Inferential tests included Multivariate Analysis of Variance (MANOVA), one-way Analysis of Variance (ANOVA) and Multiple Linear Regression (MLR) analyses, all undertaken in accordance with Tabachnick and Fidell's (2007) guidelines. Five cases were excluded from the analysis as they constituted multivariate outliers as defined by extreme Mahalanobis distances.

The Theory of Reasoned Action asserts that a person's behavioral intention depends both on the person's attitude about the behavior and upon subjective norms, and expresses an equality between behavioral intention and the weighted sum of attitude and subjective norm, that is: behavioral intention

$$
=W_{1}(\text { attitude })+W_{2}(\text { subjective norm })
$$

$W_{1}$ and $W_{2}$ are empirically derived weights, and behavioral intention is the intention to be physically active, and is a composite variable calculated from four items asking questions such as "I intend to participate in activity as much as I can each week". 


\section{Results}

The overall integrity of the scales was satisfactory, and a missing data analysis revealed that less than half a percent of data was missing. The average reported duration of PA was 9.2 hours per week (see Table 1(a)). Means $(M)$, standard deviations $(S D)$, and Cronbach's alphas $\left(\alpha_{c}\right)$ for the five ATPAS subscales are presented in Table 1(b). Mean scores on each of the DASS-42 subscales (see Table 1(c)) are comparable to those obtained from normal adult populations and lower than reported clinical norms (Lovibond \& Lovibond, 1995). Principal components analysis (PCA) undertaken on each of the three DASS subscales all returned single-factor solutions. The internal consistency of the DASS-42 has been reported elsewhere as $\alpha_{c}=.71$ for depression, $\alpha_{c}=.79$ for anxiety and $\alpha_{c}=.81$ for stress (Brown, Chorpita, Korotitsch, \& Barlow, 1997), and the estimates reported in Table 1c compare favorably with these. A PCA performed on the RSES confirmed its unidimensionality, and its internal consistency was sufficiently high $\left(\alpha_{c}=.89\right)$. The sample mean was 20.63 (Table 1(d)) and thus above the cut-off score of 15 , below which self-esteem is considered to be low (Rosenberg, 1965).

Zero-order correlation statistics (Pearson's $r$ ) were calculated to assess the relationship between psychological well-being (self-esteem, lack of depression, anxiety, and stress) and influences to PA as measured using the ATPAS (Table 2). There is no statistical evidence indicating a relationship between attitudes towards PA and psychological well-being $(p>.05)$. Statistically significant moderate $(0.3-0.6)$ positive correlations were noted between the four psychological well-being measures and barriers to PA, along with a number of smaller $(<.3)$ correlations.

To reduce the experiment-wide error rate, a simple MANOVA was conducted with PAQ group as the between-groups factor and the four latent psychological well-being variables constituting the dependent variables (DVs). Small to medium correlations existed between the four DVs at each of three PAQ levels, and a Bartlett's test of sphericity $\left(\chi^{2}(9)=703.251, p\right.$ $<.001)$ and a Box's M test of equality of covariance matrix $(F$ $=.85, p=.653)$ further confirmed the viability of a MANOVA. There was a small but significant multivariate effect of the grouped DVs in relation to PAQ grouping (Wilks Lambda $=.867, F(8,266)=2.464, p=.014)$, indicating that psychological well-being is related to group membership. Levene's tests of equality of variances were then performed prior to conducting univariate $F$ tests. For each of the four DVs the null hypothesis that the within-groups variability is equitable across the three PAQ groups was supported $(p>.100)$. The univariate $F$ tests's revealed significant differences across the three PAQ groups for depression $(F(2,136)=4.625, p=.011)$ and anxiety $(F(2,136)=$ $4.625, p<.001)$, but not for stress $(F(2,136)=2.609, p=.077)$ or self-esteem $(F(2,136)=.561, p=.572)$. Subsequent posthoc tests employing Bonferroni inequalities showed that those in the relatively inactive group (i.e., PAQ1) reported significantly higher levels of depression than those in the highly active group $(p=.014)$ but not those in the relatively active group $(p=.071)$. Additionally, those in the relatively inactive group had a significantly higher self-reported anxiety mean than both the relatively active $(p=.003)$ and highly active $(p=.026)$ PAQ groups.

Five influences on PA, namely attitude, health consciousness, significant others, priority, and barriers, were examined for associations with PAQ category. A 3 (PAQ group) $\times 5$ (influences) MANOVA was undertaken to examine the association between the grouped DVs (i.e., influences) and PAQ group. Small but significant correlations existed between the five DVs at each of three PAQ levels. Two indices, the Bartlett's test of sphericity $\left(\chi^{2}(14)=158.367, p<.001\right)$ and Box's M $(F=19.81$, $p=.949)$, confirmed that the data satisfied the homogeneity of covariance assumption. The MANOVA revealed a significant multivariate effect (Wilks Lambda $=.846, F(10,264)=2.303, p$ $=.013)$. Following non-significant Levene's tests a battery of

Table 1.

Summary statistics for the a) NZPAQ-SF; b) ATPAS; c) DASS-42; and d) RSES. Participants are divided into three activity groups: relatively inactive ( $<2.5$ hours per week; PAQ1), relatively active (2.5 - 4.9 hours per week; PAQ2), and highly active ( $>5$ hours per week; PAQ3).

\begin{tabular}{|c|c|c|c|c|c|c|c|c|c|c|c|}
\hline \multirow[b]{2}{*}{ Scale } & \multirow[b]{2}{*}{ \# of items } & \multirow[b]{2}{*}{ Overall $\alpha_{c}$} & \multicolumn{3}{|c|}{ PAQ $1(n=40)$} & \multicolumn{3}{|c|}{ PAQ $2(n=27)$} & \multicolumn{3}{|c|}{ PAQ $3(n=72)$} \\
\hline & & & $M$ & $S D$ & Scale $\alpha_{c}$ & $M$ & $S D$ & Scale $\alpha_{c}$ & $M$ & $S D$ & Scale $\alpha_{c}$ \\
\hline \multicolumn{12}{|c|}{ (a) NZPAQ-SF } \\
\hline Physical activity & 9 & - & 1.118 & .819 & - & 3.629 & .614 & - & 15.305 & 13.962 & - \\
\hline \multicolumn{12}{|c|}{ (b) ATPAS } \\
\hline Health consciousness & 11 & .718 & 46.878 & 4.905 & .765 & 49.557 & 4.388 & .642 & 49.384 & 4.704 & .724 \\
\hline Attitudes & 12 & .724 & 46.878 & 4.924 & .706 & 44.740 & 4.034 & .667 & 44.945 & 4.539 & .735 \\
\hline Significant others & 7 & .816 & 21.512 & 4.739 & .691 & 21.667 & 4.574 & .799 & 21.534 & 5.009 & .808 \\
\hline Priority & 5 & .721 & 14.049 & 3.420 & .623 & 12.815 & 3.235 & .647 & 49.384 & 3.479 & .768 \\
\hline Barriers & 8 & .681 & 23.400 & 4.499 & .574 & 20.296 & 5.179 & .695 & 19.425 & 4.551 & .636 \\
\hline \multicolumn{12}{|c|}{ (c) DASS-42 } \\
\hline Anxiety & 14 & .754 & 2.415 & 1.171 & .802 & 1.574 & 1.002 & .803 & 1.647 & .959 & .657 \\
\hline Stress & 14 & .869 & 9.195 & 7.527 & .880 & 6.037 & 5.488 & .841 & 6.959 & 5.927 & .861 \\
\hline \multicolumn{12}{|c|}{ (d) RSES } \\
\hline Self esteem & 10 & .870 & 20.875 & 5.312 & .843 & 19.593 & 5.645 & .878 & 19.972 & 5.192 & .884 \\
\hline
\end{tabular}


Table 2.

Zero-order correlations for indices of psychological well-being (column variables) and influences of PA (row variables).

\begin{tabular}{ccccc}
\hline & Depression & Anxiety & Stress & Self-esteem \\
\hline Attitudes & -.107 & .05 & .013 & -.099 \\
Health consciousness & $-.196^{*}$ & .035 & -.133 & -.124 \\
Significant others & $.175^{*}$ & .132 & $.227^{* *}$ & $.264^{* *}$ \\
Priority & $.226^{* *}$ & $.179^{*}$ & .135 & $.178^{*}$ \\
Barriers & $.392^{* *}$ & $.248^{* *}$ & $.333^{* *}$ & $.339^{* *}$ \\
\hline
\end{tabular}

${ }^{*} p<.05$ (2-tailed); ${ }^{* *} p<.001$ (2-tailed).

five univariate $F$ tests were undertaken with appropriate alpha adjustments. For three of the five DVs, attitude towards $P A$ $(F(2,136)=4.043, p=.02)$, priority $(F(2,136)=4.111, p$ $=.018)$, and barriers to $P A(F(2,136)=9.299, p<.001)$, there were significant differences across PAQ groups. Subsequent univariate pairwise comparisons applying Bonferroni procedures revealed that the highly active PAQ group had a more positive attitude towards PA than the relatively inactive group $(p=.029)$, and also placed a higher priority on PA $(p=.014)$. In terms of perceived barriers the relatively inactive group reported more barriers to activity than either of the relatively active $(p=.026)$ or highly active $(p<.001)$ PAQ groups, but no significant differences were noted between the relatively active and highly active groups.

Table 3 displays the results of four simultaneous multiple linear regression analyses undertaken after screening for normality, linearity, homoscedasticity, and independence of residuals. The criterion variable was behavioral intention, and four Theory of Reasoned Action models were tested: the three PAQ groups and also pooled data. All four models returned acceptable overall fits: relatively inactive $(F(2,38)=29.67, p$ $<.001)$, relatively active $(F(2,24)=5.332, p<.012)$, highly active $(F(2,70)=18.586, p<.001)$, and pooled data $(F(2,138)$ $=56.54, p<.001)$. Table 3 reports both unstandardized $(B)$ and standardized coefficients $(\beta)$ along with a variance accounted for statistic $\left(R^{2}\right)$. The sub-scales, attitudes towards $P A$ and significant others were the independent variables, and in a linear combination were used to predict behavioral intention (see Equation (1)).

For the relatively inactive group, the two independent variables effectively account for the variance in behavioral intention. Note that for the standardized beta coefficients attitudes towards $P A$ is significantly different from zero across the four regression analyses, whereas the significant others variable is not significant. This implies that attitudes towards $P A$ can be used to predict behavioral intention, but significant others does not possess such utility. For the pooled data, the zero-order correlation between behavioral intention and actual PA was small but also significant $(r=.206, p=.014)$.

\section{Discussion}

Approximately $29 \%$ of participants surveyed engaged in less than 2.5 hours of at least moderately intense PA per week. However, $19.5 \%$ of those surveyed were relatively active, participating in 2.5 to 4.9 hours of activity per week, and $52 \%$ were highly active, with five or more hours of activity per week. These results echo previous research undertaken in New Zealand, which showed that the proportion of participants who were physically active were $70 \%$, while $30 \%$ of adults were insufficiently active (SPARC, 2003). Thus, PA in adolescent New Zealand females may possibly be indistinguishable from adult levels.

The small but significant association between PA and two dimensions of psychological well-being (i.e., depression and anxiety) mirrors an emerging body of evidence indicating that, for both adolescents and adults, increased PA is positively associated with better mental health (Sallis et al., 2000; Teychenne, Ball, \& Salmon, 2008). Sallis et al. (2000) report an inverse relationship between depression and adolescents' levels of PA. Reviews of the literature found, for adults, strong evidence for a positive relationship between levels of occupational and leisure time PA and reduced symptoms of depression (Dunn et al., 2001; Fox, 1999). Additionally, our results support previous findings that PA is associated with a reduction in anxiety (Fox, 1999). Social cognitive and self-efficacy theory (Bandura, 1986, 1997) suggest that the relationship between PA and mental health is mediated by feelings of self-efficacy and self-concept. Annesi (2005) states that, when a person senses accomplishment in a physical task, self-concept improves and better coping with psychological stressors ensues. This, in turn, would improve mood and feelings of overall well-being. However, our finding of no association between PAQ grouping and stress suggests that PA may not function as a mechanism for stress relief in adolescent females.

In relation to influencess of PA, there was an association between activity prioritization and level of PA, with those in the highly active PAQ group reporting higher mean priority scores than those in the relatively inactive and relatively active PAQ groups. This finding cannot be explained by the higher PAQ group being more health conscious, as this was statistically equivalent across the three PAQ groups. In a qualitative study of adolescent girls and barriers to PA (Kientzler, 1999), it was noted that the most frequently reported barrier to PA was conflict with other activities, and in tandem with others (Koezuka, Koo, Allison, Adlaf, Dwyer, Faulkner, \& Goodman, 2006), our data provide quantitative support for this assertion.

The lack of association between PAQ group and the influence of others was unexpected, and reports of a strong influence of others on an individual's exercising behavior (Schofield et al., 2007; Seabra et al., 2008) has not been replicated here. However, a review by Sallis et al. (2000) identified that females were not only less likely to be physically active when compared to males, but were also less influenced by significant others and role models who were endorsing PA. After puberty, girls have greater fat mass and less muscle mass than boys, and while these biological differences may, in part, account for some of the gender and developmental variation in PA, social and environmental influences may be equally or more important (Garcia, Broda, Frenn, Coviak, Pender, \& Ronis, 1995). 
Table 3.

Unstandardized and standardized coefficients for the two sub-scales attitudes to PA and significant others that make up the Theory of Reasoned Action for the three PAQ groups $(1=$ relatively inactive, $2=$ relatively active, $3=$ highly active $)$ and for the entire sample. Model fit statistics $\left(R\right.$ and $\left.R^{2}\right)$ are included.

\begin{tabular}{|c|c|c|c|c|c|c|}
\hline \multicolumn{7}{|c|}{ PAQ1 $\left(R=.781, R^{2}=.609\right)$} \\
\hline & Std. Error & Intercept & $B$ & $\beta$ & $t$ & $p$ \\
\hline Attitudes & .25 & \multirow{2}{*}{-.39} & 1.952 & 0.78 & 7.67 & $>.001$ \\
\hline Significant others & .15 & & -.031 & -.02 & -.20 & .841 \\
\hline \multicolumn{7}{|c|}{ PAQ2 $\left(R=.539, R^{2}=.290\right)$} \\
\hline & Std. Error & Intercept & $B$ & $\beta$ & $t$ & $p$ \\
\hline Attitudes & .38 & \multirow{2}{*}{-.305} & 1.249 & 0.55 & 3.32 & .003 \\
\hline Significant others & .20 & & -.156 & -.13 & -.77 & .449 \\
\hline \multicolumn{7}{|c|}{ PAQ3 $\left(R=.652, R^{2}=.425\right)$} \\
\hline & Std. Error & Intercept & $B$ & $\beta$ & $t$ & $p$ \\
\hline Attitudes & .19 & \multirow{2}{*}{-.688} & 1.329 & .66 & 6.83 & $>.001$ \\
\hline Significant others & .12 & & -.021 & -.02 & -.18 & .858 \\
\hline \multicolumn{7}{|c|}{ Pooled data $\left(R=.671, R^{2}=.442\right)$} \\
\hline & Std. Error & Intercept & $B$ & $\beta$ & $t$ & $p$ \\
\hline Attitudes & .012 & \multirow{2}{*}{-2.098} & .130 & .688 & 10.484 & $>.001$ \\
\hline Significant others & .014 & & -.014 & -.075 & -1.142 & .256 \\
\hline
\end{tabular}

Barriers to PA were associated with PAQ group, with those in the relatively inactive PAQ group reporting higher average scores on questions probing barriers to PA than either of the relatively active and highly active PAQ groups, echoing international studies (Robbins et al., 2009). One quantitative study that explored barriers to PA among adolescent girls (Robbins, Pender, \& Kazanis, 2003), reported that self-consciousness and concerns about appearance during PA was a major barrier, while lack of interest and motivation was also identified as a substantial barrier. This later finding is interesting as impaired motivation is a common symptom of depression, which varied across the three activity groups in our data.

Our results indicate that participants with more positive attitudes towards PA also engage in higher levels of actual activity. Previous studies have found that the formation of a positive attitude towards PA is an important step towards actual participation in activity (Annesi, 2005; Smoll \& Schutz, 1980), and our data appear consistent with these findings. However, an interesting finding to emerge from this study is that while there is a relationship between perceived barriers to PA and psychological well-being, no such relationship exists between attitudes and psychological well-being. The implication here is that, for female adolescents experiencing impaired psychological wellbeing, the perceived value of PA may not be in doubt, but the perceived barriers to PA may be seen as more insurmountable as the severity of the psychological impairment increases. Thus, rather than focusing interventions on attitude change, a better strategy might be to reduce actual and/or perceived barriers identified by those experiencing psychological difficulties.

The Theory of Reasoned Action (TRA), equating a person's intention to engage in PA with a linear combination of attitude and subjective norm, was successfully applied to the data. Our results clearly show that of the two TRA components, attitude has an influence on behavioral intention whilst the subjective norm component does not. The statistically significant rela- tionship we found between intention and attitude has precedence in the literature (Chatzisarantis \& Hagger, 2005; Courneya \& Friedenreich, 1999; Courneya, Vallance, Jones, \& Reiman, 2005; Shen, McCaughtry, \& Martin, 2008) and supports the notion that intentions to engage in PA can be influenced by targeting attitudes alone (Wood, 2008). The ability of the TRA to predict behavioral intention was satisfactory, with $r^{2}$ values ranging from .3 to .6. However, behavioral intention, as predicted by the model, was itself a weak predictor of actual PA. This disparity between predicted behavioral intention scores and actual PA indicates that while attitudes play a significant role in determining intentions to engage in PA, there are other factors that must be accounted for. Our data suggests a negative relationship between barriers and level of PA, and therefore measures of behavioral intention incorporating measures of perceived barriers would enhance predictive power. Though not applied in our study, the Theory of Planned Behavior (Ajzen, 1985; Madden, Ellen, \& Ajzen, 1992) is an extension of the TRA that, in addition to the attitudes and subjective norms terms that make up the TRA, contains the additional concept of perceived behavioral control, or control beliefs.

Primary limitations of this study were that the sample size was small, inhibiting analytical options, and that a self-report inventory was employed. Self-report inventories, although they are often the only practical way to measure PA levels in representative population samples, have a known tendency to overreport activity and under-report sedentary behaviors - a tendency that may be increasing because of increasing social desirability bias (SPARC, 2004). The sample limits the generalization of results, and further data need to be collected from other scholastic contexts in order to determine if the findings we report are typical. Additionally, the present study was observational, which therefore precludes inferences about cause and effect relationships, but the results are consistent with the findings from other studies indicating that PA can positively 
affect psychological well-being (Penedo \& Dahn, 2005). Furthermore, we measured behavioral intention and actual behavior on the same occasion, thus assuming that the intention to engage in PA had not changed in the recent past.

To summarize, the present study found links between levels of PA and depression and anxiety in adolescent females. Attitudes to PA was a strong predictor of an individual's intention to engage in PA, but not the actual levels of PA itself. While levels of health consciousness and attitudes to PA did not differ between the inactive and active participants, inactive participants reported higher perceived barriers. These data indicate that the most effective manner for school-based interventions aiming to increase PA may therefore be to directly target barriers rather than addressing attitudes. To this end, school districts should consider the physical education environment alongside minimal activity requirements, so that the quality of the activities serves to reduce barriers, rather than participation.

\section{REFERENCES}

Ajzen, I. (1985). From intentions to actions: A theory of planned behaviour. In J. Kuhl, \& J. Beckmann (Eds.), Springer series in social psychology (pp. 11-39). Berlin: Springer Verlag.

Annesi, J. J. (2005). Improvements in self-concept associated with reductions in negative mood in preadolescents enrolled in an after-school physical activity program. Psychological Reports, 97, 400-404.

Azar, D., Ball, K., Salmon, J., \& Cleland, V. (2008). The association between physical activity and depressive symptoms in young women: A review. Mental Health and Physical Activity, 1, 82-88. doi:10.1016/j.mhpa.2008.09.004

Bandura, A. (1986). Social foundations of thought and action: A social cognitive theory. New Jersey: Prentice-Hall.

Bandura, A. (1997). Self-efficacy: The exercise of control. New York: Freeman.

Biddle, S., \& Mutrie, N. (2008). Psychology of physical activity: Determinants, well-being, and interventions. London; New York: Routledge.

Brown, T. A., Chorpita, B. F., Korotitsch, W., \& Barlow, D. H. (1997). Psychometric properties of the Depression Anxiety Stress Scales (DASS) in clinical samples. Behaviour Research and Therapy, 35, 79-89. doi:10.1016/S0005-7967(96)00068-X

Chatzisarantis, N. L. D., \& Hagger, MS. (2005). Effects of a brief intervention based on the theory of planned behavior on leisure-time physical activity participation. Journal of Sport Exercise Psychology, 27, 470-487.

Courneya, K. S., \& Friedenreich, C. M. (1999). Utility of the Theory of Planned Behavior for understanding exercise during breast cancer treatment. Psycho-Oncology, 8, 112-122.

doi:10.1002/(SICI)1099-1611(199903/04)8:2<112::AID-PON341>3. $0 . \mathrm{CO} ; 2-\mathrm{L}$

Courneya, K. S., Vallance, J. K. H., Jones, L. W., \& Reiman, T. (2005). Correlates of exercise intentions in non-Hodgkin's lymphoma survivors: An application of the theory of planned behavior. Journal of Sport and Exercise Psychology, 27, 335-349.

Dugan, S.A. (2008). Exercise for preventing childhood obesity. Physical Medicine and Rehabilitation Clinics of North America, 19, $205-$ 216. doi:10.1016/j.pmr.2007.11.001

Dunn, A. L., Madhukak, H. T., \& O’Neal, H. A. (2001). Physical activity dose-response effects on outcomes of depression and anxiety. Medicine \& Science in Sports \& Exercise, 33, S587-S597. doi:10.1097/00005768-200106001-00027

Fishbein, M, \&. Ajzen I. (1975). Belief, attitude, intention, and behaviour: An introduction to theory and research. Reading, MA: Addison-Wesley.

Fox, K. R. (1999). The influence of physical activity on mental wellbeing. Public Health Nutrition, 2, 411-418. doi:10.1017/S1368980099000567
Garcia, A. W., Broda, M. A. N., Frenn, M., Coviak, C., Pender, N. J., \& Ronis, D. L. (1995). Gender and developmental differences in exercise beliefs among youth and prediction of their exercise behavior. Journal of School Health, 65, 213-219. doi:10.1111/j.1746-1561.1995.tb03365.x

Godin, G., \& Shephard, R. J. (1984). Normative beliefs of school children concerning regular exercise. Journal of School Health, 54, 443-445. doi:10.1111/j.1746-1561.1984.tb08909.x

Hohepa, M., Schofield, G., \& Kolt, G. (2004). Adolescent obesity and physical inactivity. New Zealand Medical Journal, 117, U1210.

Hohepa, M., Schofield, G., \& Kolt, G. (2006). Physical activity: What do high school students think? Journal of Adolescent Health, 39, 328-336. doi:10.1016/j.jadohealth.2005.12.024

Kahn, J. A., Huang, B., Gillman, M. W., Field, A. E., Austin, S. B., Colditz, G. A., \& Frazier, A. L. (2008). Patterns and determinants of physical activity in U.S. adolescents. Journal of Adolescent Health, 42, 369-377. doi:10.1016/j.jadohealth.2007.11.143

Kientzler, A. L. (1999). Fifth- and seventh-grade girls' decisions about participation in physical activity. The Elementary School Journal, 99, 391-414. doi:10.1086/461932

Koezuka, N., Koo, M., Allison, K. R., Adlaf, E. M., Dwyer, J. J. M., Faulkner, G., \& Goodman, J. (2006). The relationship between sedentary activities and physical inactivity among adolescents: Results from the Canadian Community Health Survey. Journal of Adolescent Health, 39, 515-522. doi:10.1016/j.jadohealth.2006.02.005

Lovibond, S. H., \& Lovibond, P. F. (1995). Manual for the depression anxiety stress scales (2nd ed.). Sydney: Psychology Foundation.

Mackay, L. M., Schofield, G. M., \& Schluter, P. J. (2007). Validation of self-report measures of physical activity: A case study using the New Zealand physical activity questionnaire. Research Quarterly for Exercise and Sport, 78, 189-196. doi:10.5641/193250307X13082490460904

Madden, T. J., Ellen, P. S., \& Ajzen, I. (1992). A comparison of the theory of planned behavior and the theory of reasoned action. Personality and Social Psychology Bulletin, 18, 3-9. doi: $10.1177 / 0146167292181001$

Penedo, F. J., \& Dahn, J. R. (2005). Exercise and well-being: A review of mental and physical health benefits associated with physical activity. Current Opinion in Psychiatry, 18, 189-193. doi: 10.1097/00001504-200503000-00013

Riddoch, C. J., \& Boreham, C. A. G. (1995). The health-related physical activity of children. Sports Medicine, 19, 86-102. doi:10.2165/00007256-199519020-00002

Robbins, L. B., Pender, N. J., \& Kazanis, A. S. (2003). Barriers to physical activity perceived by adolescent girls. Journal of Midwifery and Women's Health, 48, 206-212. doi:10.1016/S1526-9523(03)00054-0

Robbins, L. B., Sikorskii, A., Hamel, L. M., Wu, T.-Y., \& Wilbur, J. (2009). Gender comparisons of perceived benefits of and barriers to physical activity in middle school youth. Research in Nursing \& Health, 32, 163-176. doi:10.1002/nur.20311

Rosenberg, M. (1965). Society and the adolescent self-image. New Jersey: Princeton University Press.

Sallis, J. F., Prochaska, J. J., \& Taylor, W. C. (2000). A review of correlates of physical activity of children and adolescents. Medicine \& Science in Sports \& Exercise, 32, 963-975. doi:10.1097/00005768-200005000-00014

Sasse, S. K., Greenwood, B. N., Masini, C. V., Nyhuis, T. J., Fleshner, M., Day, H. E. W., \& Campeau, S. (2008). Chronic voluntary wheel running facilitates corticosterone response habituation to repeated audiogenic stress exposure in male rats. Stress, 11, 425-437. doi:10.1080/10253890801887453

Schofield, L., Mummery, K.W., Schofield, G., \& Hopkins, W. (2007). The association of objectively determined physical activity behavior among adolescent female friends. Research Quarterly for Exercise and Sport, 78, 9-15. doi:10.5641/193250307X13082490460175

Seabra, A. F., Mendonça, D. M., Thomis, M. A., Peters, T. J., \& Maia, J. A. (2008). Associations between sport participation, demographic and socio-cultural factors in Portuguese children and adolescents. European Journal of Public Health, 18, 25-30. 


\section{SHEPHERD ET AL}

doi:10.1093/eurpub/ckm049

Shen, B., McCaughtry, N., \& Martin, J. (2008). Urban adolescents' exercise intentions and behaviors: An exploratory study of a transcontextual model. Contemporary Educational Psychology, 33, 841858. doi:10.1016/j.cedpsych.2007.09.002

Smoll, F. L., \& Schutz, R. W. (1980). Children's attitudes toward physical activity: A longitudinal analysis. Journal of Sport Psychology, 2, 137-147.

SPARC (2003). SPARC facts: Results of the New Zealand sport and physical activity surveys (1997-2001). Wellington: SPARC. www.activenzsurvey.org.nz/Documents/sparc-facts-1997-2001.pdf

SPARC (2004). The New Zealand physical activity questionnaires: report on the validation and use of the NZPAQ-LF and NZPAQ-SF self-report physical activity survey instruments. SPARC: Wellington. http://www.activenzsurvey.org.nz/Documents/validation-report-phys ical-activity-guestionnaires.pdf
Sparks, P., Guthrie, A. A., \& Shepherd, R. (1997). The dimensional structure of the perceived behavioral control construct. Journal of Applied Social Psychology, 27, 418-438. doi:10.1111/j.1559-1816.1997.tb00639.x

Tabachnick, B. G., \& Fidell, L. S. (2007). Using multivariate statistics (5th ed.). Boston, MA: Allyn and Bacon.

Teychenne, M., Ball, K., \& Salmon, J. (2008). Physical activity and likelihood of depression in adults: A review. Preventive Medicine, 46, 397-411. doi:10.1016/j.ypmed.2008.01.009

Thompson, A. M., \& Humbert, M. L. (2003). Factors influencing physical activity and sedentary behaviors in children and youth. Medicine \& Science in Sports \& Exercise, 35, S161. doi:10.1097/00005768-200305001-00884

Wood, M. E. (2008). Theoretical framework to study exercise motivation for breast cancer risk reduction. Oncology Nursing Forum, 35, 89-95. doi:10.1188/08.ONF.89-95 